\title{
THE EFFECT OF CALIBRATION ERRORS ON SOURCE LOCALIZATION WITH MICROPHONE ARRAYS
}

\author{
Andy W.H. Khong, Mike Brookes
}

Department of Electrical and Electronic Engineering, Imperial College London, UK

\begin{abstract}
Source localization employing time-differences-of-arrival has been employed for many applications. The accuracy of source localization is limited by the errors in the time differences of arrival estimation as well as microphone position calibration errors. Because a microphone position error will affect multiple time differences of arrival, correlation between these quantities will be introduced. This work presents a new mathematical framework in which we quantify the localization performance of a microphone array in which the microphone positions are subject to such errors.
\end{abstract}

Index Terms - Acoustic source localization, Acoustic arrays, Time-differences-of-arrival

\section{INTRODUCTION}

Advances in array processing and sensor fusion have motivated the study and implementation of microphone arrays for source localization. In military applications, microphone arrays have been deployed together with sensors such as the geophone for parameter security purposes and intruder detection [1]. The use of microphone arrays in commercial applications facilitates source localization involving automatic video steering in videoconferencing for example [2].

The most popular method for source localization is the use of time-differences-of-arrival (TDOA) between the microphones [3]. The TDOA between any two microphones defines a hyperbolic function with the microphones corresponding to the foci of the hyperbola. Using several TDOA estimates, the intersection of these hyperbolae provides the source location. In practice, this intersection point can be achieved by solving a set of nonlinear equations relating to the source and the known microphone positions. The use of Taylor series expansion is commonly employed to linearize the set of nonlinear equations. Starting from an initial position, an iterative gradient descent is applied to improve the position estimates provided that the initialization point is close to the true solution. Alternative methods for source localization are presented in [4].

The main challenges in acoustic localization are TDOA errors, calibration errors and the design of array configurations. A popular method of TDOA estimation is the generalized cross correlation (GCC) [5] which estimates the time index corresponding to the peak of the cross-correlation function. Several factors influencing the accuracy of peak estimation include multipath effects, signal-to-noise ratio and wind turbulence. The presence of multipath may result in a loss of resolution and multiple peaks in the crosscorrelation [5]. These challenges have motivated much research interests especially in room environments where reverberation can be significant [6]. An adaptive solution for TDOA estimation is also

This work is supported by the Department of Trade and Industry UK, and by Thales Research and Technology (UK) Ltd as part of the "Multi-Modal System for Perimeter Security - Seismic, Acoustic, UltraWideBand" Project. presented in [7]. In addition, source localization algorithms require the knowledge of microphone positions and many localization algorithms assume the knowledge of these locations through calibration [8]. Microphone positioning calibration errors inevitably reduce the localization accuracy. Array configuration is also an important consideration when deploying microphone arrays. Due to the miniaturization of microphones, there is increasing flexibility on how microphones can be placed in, for example, personal digital assistants (PDAs) and laptops for commercial applications. These configurations include the uniform linear array (ULA) and circular array. The cross-dipole array has also been implemented for vehicular and aerial target localization [9].

The aim of this work is to quantify positioning errors through a mathematical framework taking into account (a) errors associated with time-of-arrival (TOA) that may arise from positioning errors of the microphones and (b) cross-correlation peak estimation errors. We also compare the performances of array configurations using the above framework given the constraints of having a fixed number of microphones and aperture size. Unlike previous publications such as [10], our analysis do not assume a diagonal error covariance matrix. To address this, we first express a mathematical relationship between TDOA and localization errors in Section 2. In Section 3, we present the TDOA error model and show how we derive the maximum likelihood estimator of the position deviation and its covariance matrix. This allows us to compute the localization accuracy given the position of the microphones and compensate for any errors that may arise such as described above. In Section 4, we validate our analysis through simulation and compare the positioning errors of the cross-dipole and circular microphone arrays. In addition, we provide comparisons between the analytical and simulated results. We conclude this paper in Section 5.

\section{RELATIONSHIP BETWEEN TDOA ERRORS AND POSITION DEVIATION}

We consider the case of a single source and an array of $M$ microphones. If $\mathbf{h}_{0}$ and $\mathbf{m}_{p}$ denote $D \times 1$ vectors containing the coordinates of the source and the $p^{\text {th }}$ microphone respectively, the differences between the coordinates of the source and the $p^{\text {th }}$ microphone can then be expressed as

$$
\mathrm{d}_{p}=\mathbf{h}_{0}-\mathbf{m}_{p} .
$$

Denoting $t_{0, p q}$ as the TDOA between the $p^{\text {th }}$ and $q^{\text {th }}$ microphone, we can then express

$$
t_{0, p q}=f_{p q}\left(\mathbf{h}_{0}\right)=c^{-1}\left[\left\|\mathbf{d}_{p}\right\|_{2}-\left\|\mathbf{d}_{q}\right\|_{2}\right]
$$

for $1 \leq p<q \leq M$ where $\|\cdot\|_{2}$ is the $l_{2}$-norm operator while $c$ is the speed of sound $\left(\sim 345 \mathrm{~ms}^{-1}\right)$ and $f_{p q}\left(\mathbf{h}_{0}\right)$ is the nonlinear 
function of the source position $\mathrm{h}_{0}$ defined by (2). Hence, there are a total of $N=0.5 M(M-1)$ distinct values of $t_{0, p q}$.

Let the coordinates of a point relative to $\mathbf{h}_{0}$ be $\mathbf{h}_{0}+\mathbf{h}$ where $\mathbf{h}$ is an $D \times 1$ vector containing deviations from the source position $\mathbf{h}_{0}$. Defining the superscript ${ }^{T}$ as the transposition operator, the TDOA measurements at position $\mathbf{h}_{0}+\mathbf{h}$ can be expressed as $\mathbf{t}_{0}+\mathbf{t}$ where $\mathbf{t}_{0}=\left[t_{0,12}, t_{0,13}, \ldots, t_{0,(M-1) M}\right]^{T}$ is a $N \times 1$ vector containing TDOAs defined in (2) and $\mathbf{t}=\left[t_{12}, t_{13}, \ldots, t_{M(M-1)}\right]^{T}$ is a vector containing deviations of TDOA measurements from source position $\mathbf{h}_{0}$. Defining

$$
\begin{aligned}
\mathrm{e} & =\left[e_{12}, e_{13}, \ldots, e_{M(M-1)}\right]^{T} \\
& =\mathbf{t}_{0}+\mathbf{t}-\mathbf{f}\left(\mathbf{h}_{0}+\mathbf{h}\right)
\end{aligned}
$$

as a vector containing measurement errors where $\mathbf{f}(\cdot)$ is the nonlinear function defined in (2) for all $p, q$, the TDOA measurements at position $\mathbf{h}_{0}+\mathbf{h}$ can be approximated using first order Taylor series expansion about the position $\mathbf{h}_{0}$ giving

$$
\begin{aligned}
\mathbf{t}_{0}+\mathbf{t} & =\mathbf{f}\left(\mathbf{h}_{0}+\mathbf{h}\right)+\mathbf{e} \\
& \approx \mathbf{f}\left(\mathbf{h}_{0}\right)+c^{-1} \mathbf{J} \mathbf{h}+\mathbf{e} \\
& =\mathbf{t}_{0}+c^{-1} \mathbf{J h}+\mathbf{e} \\
\Rightarrow \mathbf{t} & =c^{-1} \mathbf{J} \mathbf{h}+\mathbf{e}
\end{aligned}
$$

where

$$
\mathbf{J}=\left[\mathbf{j}_{12}, \mathbf{j}_{13}, \ldots, \mathbf{j}_{(M-1) M}\right]^{T}
$$

is the scaled Jacobian matrix of dimension $N \times D$ with column vectors $\mathbf{j}_{p q}$ containing elements that are partial derivatives of the $t_{0, p q}$ with respect to $\mathbf{h}_{0}$. We can decompose $\mathbf{J}$ by first defining an $D \times M$ matrix G

$$
\mathbf{G}=\left[\mathbf{g}_{1}, \mathbf{g}_{2}, \ldots, \mathbf{g}_{M}\right] .
$$

where each $D \times 1$ vector $\mathbf{g}_{p}=\left(\mathbf{h}_{0}-\mathbf{m}_{p}\right) /\left\|\mathbf{d}_{p}\right\|_{2}, p=1, \ldots, M$, is a unit vector pointing towards the source from microphone $p$. The scaled Jacobian matrix is then

$$
\mathbf{J}=\mathbf{K G}^{T}
$$

where the $N \times M$ matrix $\mathbf{K}$ performs subtraction operation between vector pairs $\mathbf{g}_{p}$ and $\mathbf{g}_{q}$ and hence each row of $\mathbf{K}$ contains only two non-zero entries: +1 and -1 . Using (8) and (9), column vectors in $\mathbf{J}$ correspond to $\mathbf{j}_{p q}=\mathbf{g}_{p}-\mathbf{g}_{q}$.

\subsection{Structure and properties of $K$}

The $N \times M$ matrix $\mathbf{K}$ is of the form

$$
\mathbf{K}=\left[\begin{array}{ccccc}
1 & -1 & 0 & \cdots & 0 \\
1 & 0 & -1 & \cdots & 0 \\
\vdots & \ddots & \ddots & \ddots & \vdots \\
0 & \cdots & \cdots & 1 & -1
\end{array}\right]
$$

We note that each column of $\mathbf{K}$ contains exactly $M-1$ non-zero entries corresponding to the $M-1$ equations that involve the corresponding microphone. For any pair of columns, only one row is non-zero entry in both columns and it has opposite signs in the two columns. It follows from this that all diagonal elements of $\mathbf{K}^{T} \mathbf{K}$ equal $M-1$ and that all off-diagonal elements equal -1 , i.e.,

$$
\mathbf{K}^{T} \mathbf{K}=M \mathbf{I}-1 \mathbf{1}^{T}
$$

where 1 denotes an $M \times 1$ vector of 1 's and $\mathbf{I}$ denotes an $M \times M$ identity matrix. It is also convenient to note that

$$
\mathbf{K} \mathbf{1}=\mathbf{0} \Rightarrow \mathbf{K} \mathbf{K}^{T} \mathbf{K}=\mathbf{K}\left(M \mathbf{I}-\mathbf{1 1}^{T}\right)=M \mathbf{K} .
$$

\subsection{Maximum likelihood estimator}

We wish to estimate the position deviation $h$. We assume that the TDOA measurement error vector e has a normal distribution with zero mean and $N \times N$ covariance matrix $\mathbf{R}_{\mathrm{e}}=E\left\{\mathrm{ee}^{T}\right\}$, i.e., e $\sim$ $\mathcal{N}\left(\mathbf{e} ; 0, \mathbf{R}_{\mathrm{e}}\right)$ where $E\{\cdot\}$ is defined as the expectation operator. The probability of e given $\mathbf{h}$ is then $p(\mathbf{e} \mid \mathbf{h})=\mathcal{N}\left(\mathbf{t}-c^{-1} \mathbf{J} \mathbf{h} ; 0, \mathbf{R}_{\mathbf{e}}\right)$. We then obtain the maximum likelihood estimate $\widehat{h}$ by maximizing $p(\mathbf{e} \mid \mathbf{h})$ with respect to $\mathbf{h}$ and setting the differential to zero giving

$$
\begin{aligned}
\mathbf{0}_{N \times 1} & =\frac{d}{d \mathbf{h}}\left(\mathbf{t}-c^{-1} \mathbf{J h}\right)^{T} \mathbf{R}_{\mathbf{e}}^{-1}\left(\mathbf{t}-c^{-1} \mathbf{J h}\right) \\
& =-2 c^{-1} \mathbf{J}^{T} \mathbf{R}_{\mathbf{e}}^{-1}\left(\mathbf{t}-c^{-1} \mathbf{J} \widehat{\mathbf{h}}\right) \\
\Rightarrow \widehat{\mathbf{h}} & =c\left[\mathbf{J}^{T} \mathbf{R}_{e}^{-1} \mathbf{J}\right]^{-1} \mathbf{J}^{T} \mathbf{R}_{\mathbf{e}}^{-1} \mathbf{t}
\end{aligned}
$$

where $0_{N \times 1}$ is the $D \times 1$ null vector and $\widehat{\mathrm{h}}$ is the maximum likelihood estimator of $\mathbf{h}$. We are interested in determining the statistical properties of positioning errors, i.e., the variance of the deviations from the true position $\mathbf{h}_{0}$. This can be obtained by setting $\mathbf{h}=0_{D \times 1}$ giving $\mathbf{t}=\mathrm{e}$ with a PDF of $\mathcal{N}\left(\mathbf{e} ; \mathbf{0}, \mathbf{R}_{\mathrm{e}}\right)$. The covariance matrix of $\widehat{\mathbf{h}}$ is then given by

$$
\begin{aligned}
\mathbf{R}_{\widehat{\mathbf{h}}} & =\widehat{\mathbf{h}} \widehat{\mathbf{h}}^{T} \\
& =c^{2}\left(\mathbf{J}^{T} \mathbf{R}_{\mathrm{e}}^{-1} \mathbf{J}\right)^{-1} \mathbf{J}^{T} \mathbf{R}_{\mathrm{e}}^{-1} \mathbf{R}_{\mathrm{e}} \mathbf{R}_{\mathrm{e}}^{-1} \mathbf{J}\left(\mathbf{J}^{T} \mathbf{R}_{\mathrm{e}}^{-1} \mathbf{J}\right)^{-1} \\
& =c^{2}\left(\mathbf{J}^{T} \mathbf{R}_{\mathrm{e}}^{-1} \mathbf{J}\right)^{-1}
\end{aligned}
$$

and hence our aim is to compute $\widehat{\mathbf{h}}$ and $\mathbf{R}_{\widehat{\mathbf{h}}}$ in order to quantify the localization performance of microphone arrays given the microphone positions subject to errors.

\section{TDOA ERROR MODEL}

We assume that the TDOA errors, e, arise from two zero mean sources (a) an independent TOA error at each microphone, $v_{m}, m=$ $1, \ldots, M$ and (b) an independent TDOA evaluation error, $b_{n}, n=$ $1, \ldots, N$, for each of the $N$ equations. The independent TOA errors encompass both microphone positioning errors that may arise due to calibration errors and also errors due to sound propagation delay uncertainties. The TDOA evaluation errors are a function of the signal and noise spectra and lower bounds are given in [11]. The $N$ errors due to (a) and (b) can then be expressed jointly as

$$
\mathbf{e}=\mathbf{b}+\mathbf{K v} \text {, }
$$

where $\mathbf{b}=\left[b_{1}, \ldots, b_{N}\right]^{T}$ and $\mathbf{v}=\left[v_{1}, \ldots, v_{M}\right]^{T}$. If we take the covariance matrices of $\mathbf{b}$ and $\mathbf{v}$ to be $\sigma_{b}^{2} \mathbf{I}$ and $\mathbf{S}=\operatorname{diag}\{\mathbf{v}\}$ respectively we obtain

$$
\mathbf{R}_{\mathbf{e}}=E\left\{\mathbf{e} \mathbf{e}^{T}\right\}=\sigma_{b}^{2} \mathbf{I}+\mathbf{K S}^{2} \mathbf{K}^{T},
$$

and hence we do not assume that $\mathbf{R}_{e}$ is a diagonal matrix.

\subsection{Inverse covariance of TDOA errors}

To compute $\mathbf{R}_{e}^{-1}$ for (13) and (14), we employed the matrix inversion lemma [12] giving,

$$
\begin{aligned}
\mathbf{R}_{e}^{-1} & =\sigma_{b}^{-2} \mathbf{I}-\sigma_{b}^{-2} \mathbf{K S}\left[\mathbf{I}+\sigma_{b}^{-2} \mathbf{S K}^{T} \mathbf{K S}\right]^{-1} \mathbf{S K}^{T} \sigma_{b}^{-2} \\
& =\sigma_{b}^{-2} \mathbf{I}-\sigma_{b}^{-2} \mathbf{K S}\left[\sigma_{b}^{2} \mathbf{I}+M \mathbf{S}^{2}-\mathbf{s s}^{T}\right]^{-1} \mathbf{S K}^{T} \\
& =\sigma_{b}^{-2} \mathbf{I}-\sigma_{b}^{-2} \mathbf{K S}\left[\mathbf{A}^{-1}-\mathbf{s s}^{T}\right]^{-1} \mathbf{S} \mathbf{K}^{T}
\end{aligned}
$$

where we have used (11), $\mathbf{S} \mathbf{1}=\mathbf{s}$ and

$$
\mathbf{A}=\left[\sigma_{b}^{2} \mathbf{I}+M \mathbf{S}^{2}\right]^{-1} .
$$


The inversion of $\mathbf{A}^{-1}-\mathbf{s s}^{T}$ in (16) can be achieved by invoking the matrix inversion lemma giving

$$
\left[\mathbf{A}^{-1}-\mathbf{s s}^{T}\right]^{-1}=\mathbf{A}+\frac{\mathbf{A} \mathbf{s} \mathbf{s}^{T} \mathbf{A}}{1-\mathbf{s}^{T} \mathbf{A} \mathbf{s}},
$$

from which we then obtain

$$
\mathbf{R}_{\mathbf{e}}^{-1}=\sigma^{-2} \mathbf{I}-\sigma_{b}^{-2} \mathbf{K} \mathbf{S}\left[\mathbf{A}+\frac{\mathbf{A s s}^{T} \mathbf{A}}{1-\mathbf{s}^{T} \mathbf{A} \mathbf{s}}\right] \mathbf{S K}^{T} .
$$

\subsection{Equal TOA error variances}

For the remainder of this section we consider the particular case where $\mathbf{S}=\sigma_{s} \mathbf{I}$. This allows us to make the following simplifications

$$
\begin{aligned}
\mathbf{A}= & \left(\sigma_{b}^{2}+M \sigma_{s}^{2}\right)^{-1} \mathbf{I} \\
\frac{\mathbf{A} \mathbf{s} \mathbf{s}^{T} \mathbf{A}}{1-\mathbf{s}^{T} \mathbf{A} \mathbf{s}}= & \frac{\sigma_{s}^{2}\left(\sigma_{b}^{2}+M \sigma_{s}^{2}\right)^{-2} \mathbf{1} 1^{T}}{1-M \sigma_{s}^{2}\left(\sigma_{b}^{2}+M \sigma_{s}^{2}\right)^{-1}} \\
= & \sigma_{b}^{-2} \sigma_{s}^{2}\left(\sigma_{b}^{-2}+M \sigma_{s}^{2}\right)^{-1} \mathbf{1} 1^{T} \\
\mathbf{R}_{\mathbf{e}}^{-1}= & \sigma_{b}^{-2} \mathbf{I}-\sigma_{s}^{2} \sigma_{b}^{-2} \mathbf{K}[\mathbf{A} \\
& \left.\quad+\sigma_{b}^{-2} \sigma_{s}^{2}\left(\sigma_{b}^{2}+M \sigma_{s}^{2}\right)^{-1} \mathbf{1} 1^{T}\right] \mathbf{K}^{T} \\
= & \sigma_{b}^{-2} \mathbf{I}-\sigma_{s}^{2} \sigma_{b}^{-2}\left(\sigma_{b}^{2}+M \sigma_{s}^{2}\right)^{-1} \mathbf{K} \mathbf{K}^{T}
\end{aligned}
$$

where we have employed the relationship $K 1=0$. We note that the computation of (20) does not require any matrix inversion and unlike previous works, we have not assume independent TDOA errors.

\subsection{Maximum likelihood estimator using error model}

The maximum likelihood estimator $\widehat{\mathrm{h}}$ in (13) can be found by first noting that

$$
\begin{aligned}
\mathbf{J}^{T} \mathbf{R}_{\mathrm{e}}^{-1} & =\mathbf{G} \mathbf{K}^{T}\left[\sigma_{b}^{-2} \mathbf{I}-\sigma_{s}^{2} \sigma_{b}^{-2}\left(\sigma_{b}^{2}+M \sigma_{s}^{2}\right)^{-1} \mathbf{K K}^{T}\right] \\
& =\mathbf{G}\left[\sigma_{b}^{-2}-M \sigma_{s}^{2} \sigma_{b}^{-2}\left(\sigma_{b}^{2}+M \sigma_{s}^{2}\right)^{-1}\right] \mathbf{K}^{T} \\
& =\left(\sigma_{b}^{2}+M \sigma_{s}^{2}\right)^{-1} \mathbf{J}^{T}
\end{aligned}
$$

Substituting (21) into (13), we obtain the maximum likelihood estimator of the deviation from source position

$$
\widehat{\mathbf{h}}=c\left[\mathbf{J}^{T} \mathbf{J}\right]^{-1} \mathbf{J}^{T} \mathbf{t},
$$

which is independent of $\sigma_{b}$ and $\sigma_{s}$.

\subsection{Covariance of deviation using error model}

The covariance matrix of the deviation from true sensor position given by (14) can be simplified using (21) as

$$
\begin{aligned}
\mathbf{R}_{\widehat{\mathbf{h}}} & =c^{2}\left(\mathbf{J}^{T} \mathbf{R}_{e}^{-1} \mathbf{J}\right)^{-1} \\
& =c^{2}\left(\sigma_{b}^{2}+M \sigma_{s}^{2}\right)\left[\mathbf{J}^{T} \mathbf{J}\right]^{-1}
\end{aligned}
$$

It is now apparent that the computation of $\widehat{\mathrm{h}}$ and $\mathbf{R}_{\widehat{\mathrm{h}}}$ is limited by the condition number of $\mathbf{J}^{T} \mathbf{J}$ denoted by $\chi\left[\mathbf{J}^{T} \mathbf{J}\right]$. We note that each row of $\mathbf{J}$ consists of the differences between two unit vectors pointing towards the source from two microphones as described by $\mathbf{j}_{p q}=$ $\mathbf{g}_{p}-\mathbf{g}_{q}$. Consider the case where all unit vectors point toward the same direction hence giving $\mathbf{g}_{p}=\mathbf{g}_{q}, \forall p, q$. In this situation, $\mathbf{J}=\mathbf{0}$ and consequently $\mathbf{J}^{T} \mathbf{J}$ is singular hence giving poor estimates. Such a situation exists, for example, when the source is at the endfire of a linear array.
Table 1. Coordinates of microphones used in simulations

\begin{tabular}{|c|c|c|}
\hline microphone & cross-dipole & circular \\
\hline 1 & $0.0,0.0$ & $0.35,0.35$ \\
\hline 2 & $0.5,0.0$ & $-0.23,0.45$ \\
\hline 3 & $0.0,0.5$ & $-0.49,-0.08$ \\
\hline 4 & $0.0,-0.5$ & $-0.08,-0.49$ \\
\hline 5 & $-0.5,0.0$ & $0.45,-0.23$ \\
\hline
\end{tabular}

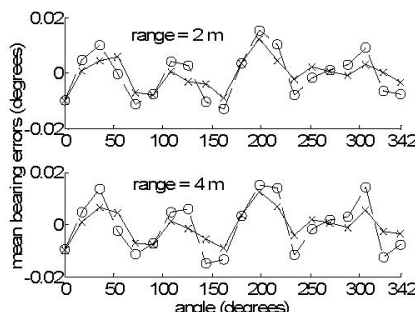

(a)

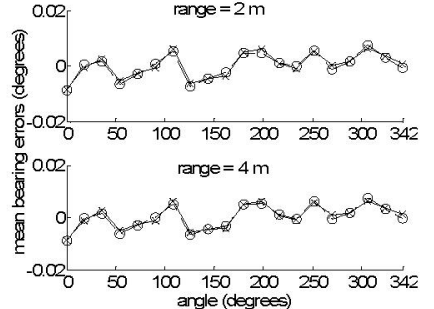

(b)
Fig. 1. Mean bearing errors for (a) cross-dipole and (b) and circular array obtained using Taylor series approximation (-०-) and "Lsqnonlin" simulation $(-x-)$.

\section{SIMULATION RESULTS}

We now provide simulation results to validate our derivations and compare the performances of array configurations in a planar case where $D=2$. We estimated source positions $\mathbf{h}_{0}+\widehat{\mathbf{h}}$ for 5000 trials using two methods (a) minimizing $\|\mathrm{e}\|^{2}$ in (3) using the lsqnonlin function in MATLAB and (b) minimizing $\|\mathrm{e}\|^{2}$ in (6) by means of (22). We constrained the number of microphones to 5 which then allow us to consider both the cross-dipole and circular arrays. For fair comparison, all microphones lie within a circle of radius $0.5 \mathrm{~m}$. Table 1 shows the coordinates of the microphones for each configuration. In these experiments, the standard deviation of the TOA for each microphone is set to $\sigma_{s}=5.79 \times 10^{-6}$ which corresponds to $2 \mathrm{~mm}$ in microphone positioning error. The independent calculation error is set to $\sigma_{b}=2.08 \times 10^{-5} \mathrm{~s}$. We analyzed the performance of each array by positioning the source at angular positions every 18 degrees for both ranges 2 and $4 \mathrm{~m}$.

Figure 1 (a) and (b) show respectively the mean bearing errors for the cross-dipole and circular array obtained using Taylor series approximation denoted by (-o-) and Lsqnon lin denoted by $\left(-X_{-}\right)$. It can be seen that the mean range errors obtained using the first order Taylor series expansion are close to that of the simulated errors. We also note that the mean bearing errors are within $0.02^{\circ}$ for both the cross-dipole and circular array configuration.

Figure 2 shows the standard deviations of the bearing errors for (a) the cross-dipole and (b) the circular array. As before, results are obtained using Taylor series approximation denoted by (-o-) and Lsqnonlin denoted by $(-x-)$. In addition, we computed the theoretical standard deviation for the bearing errors using (23) denoted by $(-\bullet-)$. These results show no significant differences between results obtained using Taylor series approximation and simulation for a source range of $2 \mathrm{~m}$ and hence we see that the linearized theory provides a good estimate of the position error uncertainty. The Taylor series approximation over estimates the standard deviation to within $0.04^{\circ}$ and $0.005^{\circ}$ for a source range of $4 \mathrm{~m}$ for the cross-dipole and circular array respectively. Comparing Figs. 1 and 2, we note that the standard deviations of the bearing errors dominate over the mean 


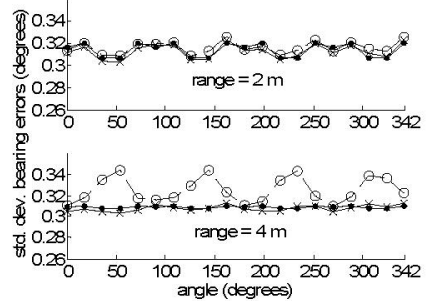

(a)

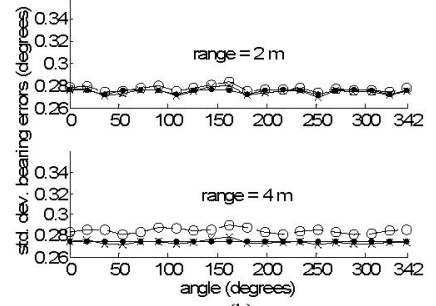

(b)
Fig. 2. Standard deviation of bearing errors for (a) cross-dipole and (b) circular array obtained using Taylor series approximation (-०-), "Lsqnonlin" simulation (-X-) and Eqn. (23) (-•-).

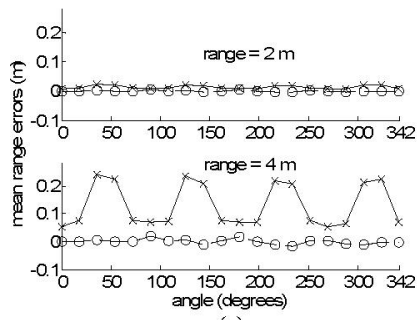

(a)

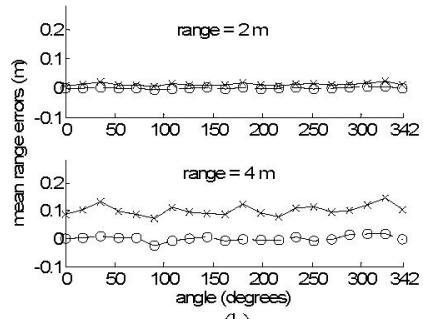

(b)
Fig. 3. Mean range errors for (a) cross-dipole and (b) circular array obtained using Taylor series approximation (-o-) and "Lsqnonlin" simulation (- $\times$-).

bearing errors for both array configurations considered. Comparing Figs. 2 (a) and (b), the standard deviation of the bearing errors for the circular array is lower than that for the cross-dipole array.

Figure 3 show the mean range errors for the (a) cross-dipole and (b) circular array. As before, we note the closeness between the simulated results and results generated using the first order Taylor series approximation given in (5) for a source range of $2 \mathrm{~m}$. The Taylor series approximation gives an approximate zero mean range error for both array configurations but results obtained using Lsqnonlin shows that the range is in fact consistently overestimated. We also note that the circular array provides lower mean range errors compared to the cross-dipole array.

Figures 4 (a) and (b) show respectively the standard deviation of range errors for the cross-dipole and circular array. Similar to Fig. 2, we included the analytical estimate given by (23). We observe that the standard deviation of range errors increases with range for both the cross-dipole and circular array. Results for the Taylor series approximation showed that the standard deviation for the range errors is lower than that using the Lsqnon $\mathrm{l}$ in function for a source range of $4 \mathrm{~m}$. In addition, we note that the circular array has a lower standard deviation for the range errors than the cross-dipole array configuration.

\section{CONCLUSION}

We presented a new mathematical framework to evaluate the performance of source localization for acoustic arrays. This analysis do not assume independent TDOA errors between the microphones and we evaluate the mean and standard deviations of positioning errors by employing the matrix inversion lemma. It has been shown that the errors between the true source position and estimated position for both Taylor series expansion and simulation increase with range for both the cross-dipole and circular arrays. Simulation results have

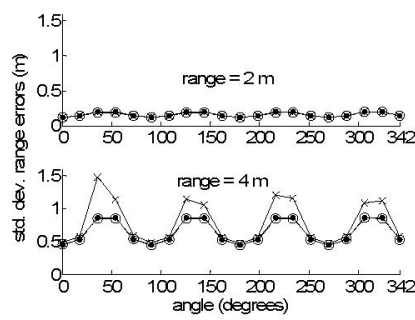

(a)

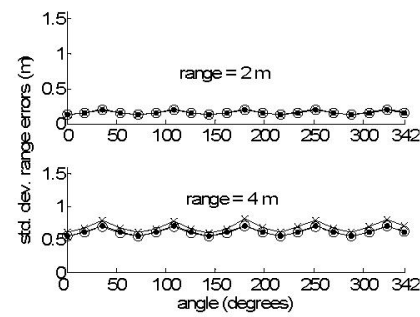

(b)
Fig. 4. Standard deviation of range errors for (a) cross-dipole and (b) circular array obtained using Taylor series approximation (-०-), "Lsqnonlin" simulation $(-\times-)$ and Eqn. (23) (-๑-).

verified our analysis and have shown that the Taylor series expansion provides a good estimate of the positioning error uncertainty. We observe from these results that although their best performances are similar, the worst-case performance of the circular array is much better than that of the cross-dipole array.

\section{REFERENCES}

[1] G. P. Succi, D. Clapp, R. Gampert, T. K. Pedersen, and G. Prado, "On the design of a small passive sensor for locating vehicles, footsteps and gunshots," in Proc SPIE Conf. on Enabling Technologies for Law Enforcement and Security, vol. 4232, Boston, 2001, pp. 367-376.

[2] Y. Huang, J. Benesty, and G. W. Elko, "Microphone arrays for video camera steering," in Acoustic Signal Processing for Telecommunication, S. L. Gay and J. Benesty, Eds. Kluwer, 2000, ch. 11, pp. 239 259

[3] K. C. Ho and W. Xu, "An accurate algebraic solution for moving source location using TDOA and FDOA measurements," IEEE Trans. Signal Processing, vol. 52, no. 9, pp. 2453-2463, Sept. 2004.

[4] P. Stoica and J. Li, "Source localization from range-difference measurements," IEEE Signal Proc Mag, vol. 23, no. 6, pp. 63-66, Nov. 2006.

[5] C. Knapp and G. Carter, "The generalized correlation method for estimation of time delay," IEEE Trans Acoustics Speech and Signal Processing, vol. 24, no, 4, pp. 320-327, Aug. 1976.

[6] J. Chen, J. Benesty, and Y. Huang, "Robust time delay estimation exploiting redundancy among multiple microphones," IEEE Trans Speech and Audio Processing, vol. 11, no. 6, pp. 549-557, Nov. 2003.

[7] N. Madhu and R. Martin, "Robust speaker localization through adaptive weighted pair TDOA (AWEPAT) estimation," in Proc Interspeech, 2005, pp. 2341-2344.

[8] V. C. Raykar and R. Duraiswami, "Automatic position calibration for multiple microphones," in Proc. IEEE Int. Conf. Acoustics Speech Signal Processing, vol. 4, 2004, pp. 69-72.

[9] K. Lo and B. Ferguson, "Broadband passive acoustic technique for target motion parameter estimation," IEEE Trans Aerospace and Electronic Systems, vol, 36, no. 1, pp. 163-175, Jan. 2000.

[10] I. Kadar, "Optimum geometry selection for sensor fusion," in Proc SPIE Signal Processing, Sensor Fusion, and Target Recognition VII, vol. 3374, Jul. 1998, pp. 96-107.

[11] A. Quazi, "An overview on the time delay estimate in active and passive systems for target localization," IEEE Trans Acoustics Speech and Signal Processing, vol. 29, no. 3, pp. 527-533, 1981.

[12] D. J. Tylavsky and G. R. Sohie, "Generalization of the matrix inversion lemma," Proc. IEEE, vol. 74, no. 7, pp. 1050-1052, July 1986. 